\title{
Different Pattern of Clinical Deficits in Stroke Mimics Treated with Intravenous Thrombolysis
}

\author{
Hakan Sarikaya Murat Yilmaz Andreas R. Luft Andreas R. Gantenbein \\ Department of Neurology, University Hospital of Zurich, Zurich, Switzerland
}

\section{Key Words}

Intravenous thrombolysis - Ischemic stroke - Stroke mimics . Adverse events

\begin{abstract}
Background: Guidelines recommend intravenous thrombolysis (IVT) to be applied as early as possible in ischemic stroke (IS), while clinical presentation is often assessed by using the National Institutes of Health Stroke Scale (NIHSS). However, diagnostic workup under time pressure bears the risk of misdiagnosis. Little is known about whether NIHSS could help to differentiate between IS and stroke mimics (SM) in patients being evaluated for IVT. Methods: Prospectively collected data of 326 consecutive patients treated with IVT were analyzed. Baseline characteristics and NIHSS subscores were compared between SM and IS. Results: Among 326 patients, 23 (7\%) had a final diagnosis other than IS. Age and vascular risk factors were comparable in both groups. Patients with SM less often had oculomotor disturbance (0 vs. $37 \%, p<0.001$ ), dysarthria (9 vs. $51 \%, p<0.001$ ), hemineglect ( 0 vs. $30 \%, p<0.01$ ), hemianopia ( 0 vs. $22 \%, p<0.01$ ) and facial palsy ( 33 vs. $70 \%, p<0.01$ ). On the other hand, global aphasia without hemiparesis was more prevalent in SM patients ( 43 vs. $6 \%, p<0.001$ ). Conclusion: Our study suggests that patients with SM undergoing IVT present with a different pattern of clinical deficits than patients with IS.
\end{abstract}

Copyright $\odot 2012$ S. Karger AG, Basel
(C) 2012 S. Karger AG, Basel 0014-3022/12/0686-0344\$38.00/0

Fax +41613061234 E-Mail karger@karger.ch www.karger.com

\section{Introduction}

Patients presenting with an acute onset of neurological symptoms need to be quickly evaluated because they may have a stroke for which immediate treatment is mandatory. Intravenous thrombolysis (IVT) can only be delivered within $4.5 \mathrm{~h}$ of symptom onset, with the likelihood of good outcome decreasing with time to treatment [1]. Therefore, history and examination remain short and focused in the emergency setting, while clinical stroke severity is often assessed using the National Institutes of Health Stroke Scale (NIHSS) [2]. Acute brain imaging is often restricted to computed tomography (CT), which can exclude intracranial hemorrhage but, in most cases, cannot prove ischemia. These constraints pose the risk of misdiagnosis and administering IVT to patients who present with symptoms suggestive of ischemic stroke (IS) but finally have a different diagnosis at discharge (so-called 'stroke mimic', SM). It has not been evaluated whether patients with SM differ from those with IS in NIHSS subscores, which could help physicians to suspect SM clinically. Furthermore, little is known about the frequency of SM in the extended 4.5-hour treatment time window. We aimed to assess the frequency, clinical characteristics and the risk for adverse events in SM patients treated with IVT within $4.5 \mathrm{~h}$ after symptom onset. 


\section{Patients and Methods}

We analyzed data collected prospectively as part of the $\mathrm{Zu}$ rich Observational Registry of Rehabilitation Outcomes (ZOR$\mathrm{RO})$. In total, 326 consecutive patients who were treated with intravenous recombinant tissue plasminogen activator (r-tPA) in the University Hospital Zurich from October 2008 to May 2011 were included. SM was diagnosed when all of the following 3 criteria were met: (1) clinical condition at admission suggesting acute IS; (2) normal diffusion-weighted magnetic resonance imaging (MRI) of the brain, and (3) specific diagnosis at discharge other than IS. The specific diagnosis was based on the clinical course and additional investigations if needed (e.g. characteristic findings in electroencephalography; history of migraine, epilepsy or conversion disorder; specific laboratory or imaging findings). Patients with normal diffusion-weighted MRI but unclear diagnosis at hospital discharge were excluded, as were patients with clinically probable stroke or transient ischemic attack and normal MRI. Normal neuroimaging in the setting of unknown cause as well as probable stroke or transient ischemic attack was excluded from the definition. Baseline investigations included neurologic and physical examination, assessment of stroke severity at admission using the NIHSS [2], routine blood analysis, 12-lead electrocardiography (ECG) and brain CT. The following variables were prospectively collected: age, gender, baseline NIHSS score, vascular risk factors according to predefined criteria [3], time to treatment and history for coronary heart disease or prior IS, and the NIHSS subscores. Thrombolysis was applied according to current guidelines with $\mathrm{r}$-tPA $0.9 \mathrm{mg} / \mathrm{kg}$ to a maximum of $90 \mathrm{mg}, 10 \%$ of the total dose given as a bolus and the remaining dose over the next hour [4]. Based on the results of the European Cooperative Acute Stroke Study (ECASS) III, we regularly extended the time window for all IVT to $4.5 \mathrm{~h}$ after symptom onset from October 2008 onwards [5]. All patients treated with IVT were admitted to intermediate or intensive care units for at least $24 \mathrm{~h}$.

\section{Outcome Parameters}

All intracranial hemorrhages were ascertained on follow-up CT or MRI routinely performed at $24 \mathrm{~h}$ after IVT and additional scans in case of clinical deterioration. Symptomatic intracranial hemorrhage (sICH) was defined as any intracranial bleed temporally related to a neurological deterioration [6]. Furthermore, we assessed the NIHSS and modified Rankin Scale (mRS) score at discharge in patients with a final diagnosis of SM $[2,7]$.

\section{Literature Search}

Two independent observers (A.R.G., H.S.) performed a detailed literature search in the PubMed, Embase and Cochrane databases for any reports on IVT in patients presenting with SM (search period defined from January 1995 to August 2011). First, we searched for the terms ('stroke mimic' OR 'stroke misdiagnosis') AND ('thrombolysis', 'alteplase' OR 'rtPA'). We also screened reference lists of all retrieved reports. There were no restrictions regarding language or publication status.

Statistical Analysis

Baseline and demographic data were examined with descriptive statistics. For the comparison of the group of SM versus IS, Fisher's exact test was used for the dichotomous variables, and the
Wilcoxon rank-sum test was used for the continuous variables. Significance was assumed at $\mathrm{p}<0.05$. All tests were performed with the statistical software R 2.13 (2011).

\section{Results}

\section{Study Population}

Within the study period, a total of 326 patients with suspected IS were treated with IVT. Of these, 23 (7\%) patients (13 males, 10 females) with a mean age of 63 years (range 26-86) presented with SM. There were no significant differences in demographic characteristics and vascular risk factors as compared to patients with IS (table 1). Stroke severity at admission was less severe in patients with SM (NIHSS 6 vs. 10, p = 0.003) (table 1). Furthermore, on average, patients with SM were treated 52 min later than patients with IS (208 vs. $156 \mathrm{~min}$ ). Table 2 shows a detailed description of clinical and imaging findings in the 23 patients with SM. Before thrombolysis, 21 patients underwent neuroimaging by CT and CT angiography (CTA), 1 patient (No. 1) by CT without contrast agent and 1 patient (No. 8) by CT, CTA and perfusion CT. All CTA and the perfusion CT were normal. MRI was performed in all 23 patients with SM at $24 \mathrm{~h}$ after IVT, and the diffusion-weighted imaging sequences were normal in all of them. SM were most frequently diagnosed as epileptic seizures $(\mathrm{n}=14)$, migraine with aura $(n=3)$ and conversion disorder $(n=3)$. The remaining 3 patients had less frequent causes for SM: hypoglycemia (blood glucose of $2.6 \mathrm{mmol} / \mathrm{l}$ at admission), sinusitis with systemic infection and multiple sclerosis with a demyelinating lesion in the cervical spinal cord.

We assessed the NIHSS subgroups in the two cohorts at admission. As compared to IS, no patient with SM presented with oculomotor disturbance (0 vs. $37 \%, \mathrm{p}=$ 0.0001 ), hemianopia ( 0 vs. $22 \%, \mathrm{p}=0.0093$ ) or hemineglect ( 0 vs. $30 \%, \mathrm{p}=0.0013$ ). Dysarthria and facial palsy were less often observed in these patients ( 9 vs. $51 \%, \mathrm{p}=$ 0.0003 and 33 vs. $70 \%, p=0.0012$, respectively). Furthermore, a marginal difference was observed in the frequency of arm paresis, which was slightly higher in the IS group (78 vs. $52 \%, \mathrm{p}=0.0166$ ). On the other hand, no significant differences were evident between the SM and IS groups when comparing common stroke findings such as leg paresis (48 vs. $66 \%, \mathrm{p}=0.0984$ ), sensory disturbances (38 vs. $53 \%, \mathrm{p}=0.252$ ), problems in answering questions ( 57 vs. $34 \%, p=0.053$ ) or performing tasks ( 24 vs. $22 \%$, $\mathrm{p}=1.0)$. However, the combined occurrence of global 
Table 1. Comparison of baseline characteristics in thrombolyzed patients with SM $(n=23)$ or IS $(n=303)$

\begin{tabular}{lccc}
\hline & SM & IS & p value \\
\hline Age, years & $63.0 \pm 19.2$ & $69.9 \pm 14.0$ & $0.19^{*}$ \\
Male gender & $13 / 23(56.5 \%)$ & $202 / 303(66.7 \%)$ & 0.36 \\
Arterial hypertension & $13 / 23(56.5 \%)$ & $209 / 296(70.6 \%)$ & 0.16 \\
Diabetes mellitus & $4 / 21(19.0 \%)$ & $41 / 262(15.6 \%)$ & 0.76 \\
Current smoking & $7 / 22(31.9 \%)$ & $60 / 270(22.2 \%)$ & 0.30 \\
History of IS & $2 / 22(9.1 \%)$ & $58 / 288(20.1 \%)$ & 0.27 \\
Coronary artery disease & $2 / 22(9.1 \%)$ & $59 / 296(19.9 \%)$ & 0.27 \\
NIHSS score & $6.43 \pm 5.21$ & $9.89 \pm 6.0$ & $0.003^{*}$ \\
OTT, min & $208.3 \pm 43.7$ & $156.1 \pm 68.0$ & $<0.001^{*}$ \\
\hline
\end{tabular}

Values are mean $\pm \mathrm{SD}$, unless otherwise indicated. OTT $=$ Onset to treatment time.

$\mathrm{p}$ values from Fisher's exact test, unless otherwise indicated ( ${ }^{*}$ Wilcoxon rank-sum test).

aphasia without hemiparesis was much more frequent in the SM group (43 vs. $6 \%, \mathrm{p}=0.00003$ ).

None of the 23 patients with SM experienced $\mathrm{sICH}$, systemic bleeding or death. At discharge, 16 (70\%) patients were without any neurological symptoms (NIHSS score 0 , mRS score 0 ), whereas 7 (30\%) patients had persisting residual deficits due to the condition that caused the acute neurological deficit but without any worsening of the symptoms after IVT.

\section{Literature Review}

Our literature search resulted in 13 reports (10 cohort studies, 3 case reports) fulfilling the predefined criteria [8-20]. These included a total of 209 patients with SM (mean frequency 6\%) treated with IVT, 200 (96\%) of them within the 3 -hour treatment time window. sICH was reported in 1 of $209(0.5 \%)$ cases [10].

\section{Discussion}

Although time constraints in the diagnostic workup and administration of IVT for patients with suspected IS are prone to misdiagnosis, this study demonstrates that IVT carries minimal risks of serious adverse events in these patients. In our cohort, $7 \%$ of thrombolyzed patients had a misdiagnosis of IS. Similar to our findings, Giraldo et al. [19] reported that 9 of 89 (10\%) patients undergoing IVT within $4.5 \mathrm{~h}$ were misdiagnosed as having IS. For the 3-hour treatment time window, SM rates of $1-13 \%$ (mean 6\%) have been reported $[8,11-14,16,18,20]$. The wide range of SM rates in these studies probably relates to several factors such as study size, definition of SM, treatment protocol and experience of the respective center. Patients with SM underwent IVT later than patients with IS in this series. In the absence of data about doorto-needle time, we do not know whether this difference was caused by a pre-hospital or an in-hospital delay, hence whether this delay was related to the uncertainty of the patient or primary/emergency physician to send the patient to the hospital or a longer diagnostic workup in the hospital. Our data suggest that age and the presence of vascular risk factors do not predict SM. In fact, epileptic seizures mimicking IS typically occurred in older patients with higher vascular risk profiles. In contrast, young patients with IS often have no cardiovascular risk factors and stroke of non-atherosclerotic etiology, e.g. due to cervical artery dissection [21].

The main strength of our study is the comparison of NIHSS subscores in patients with SM and IS. Our results show that the presence of oculomotor disturbance, hemianopia, facial palsy, dysarthria or hemineglect strongly suggests IS. In contrast, global aphasia in the absence of hemiparesis should raise the suspicion of SM, which is in line with the findings of Winkler et al. [12]. It is noteworthy that the frequency of common stroke symptoms such as aphasia, leg paresis or sensory disturbances was similarly distributed in the two groups, whereas arm paresis was slightly more often observed in IS patients. However, the latter finding may be coincidental in view of the small sample size and marginal statistical difference.

Epileptic seizure was the most common cause for SM in our cohort, followed by migraine and conversion disorder. These findings are in line with the literature [12, 14, 20]. Among less frequent etiologies, multiple sclerosis, hypoglycemia and systemic infection mimicked IS in our 


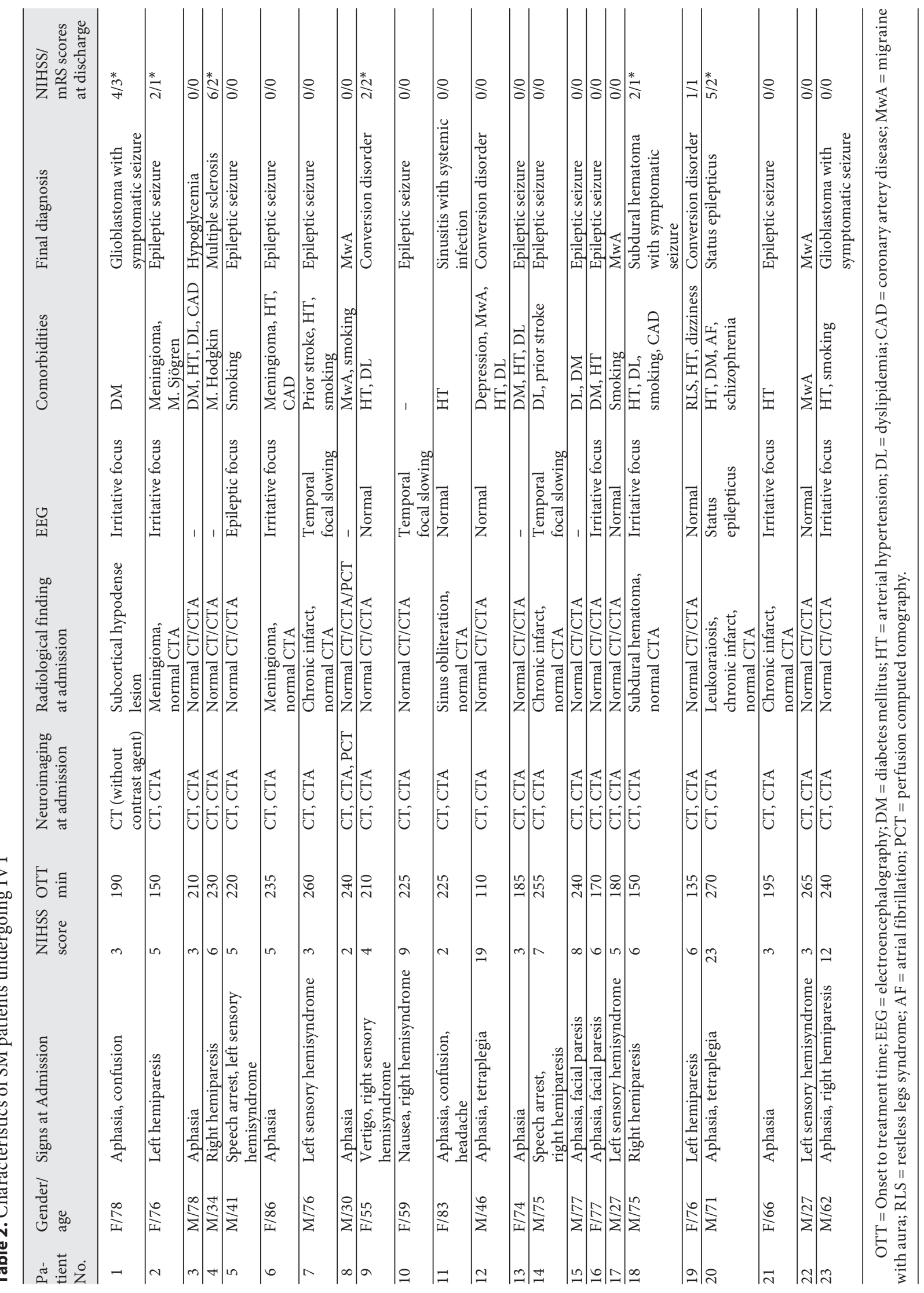


study. Perfusion CT, which has a broad availability and short processing time, may be an alternative diagnostic tool to detect epileptic seizures mimicking acute IS: while cerebral regional hyperperfusion has been reported to be characteristic for non-convulsive status epilepticus [22, 23], focal hypoperfusion in atypical vascular distributions was reported for post-ictal neurological deficits [24]. CTA was performed in 22 of 23 patients with SM and showed normal findings in all of them. Retrospectively, the obvious discrepancy between patent cerebral arteries in CTA and high NIHSS scores (e.g. patient No. 10, 12, 20 and 23) should suggest SM. Furthermore, neurologists should carefully consider the CT findings prior to thrombolysis. Reviewing the CT images, 3 patients (No. 1, 11 and 18) had subtle but visible lesions that retrospectively should have raised the suspicion of SM. In patient No. 18, treatment with alteplase was stopped $15 \mathrm{~min}$ after bolus application when a subdural hematoma was detected after reviewing the CT images.

To assess the risk of adverse events after IVT, our meta-analysis includes 232 patients with SM treated with IVT. None of the patients died. Grimm and DeAngelis [10] reported 1 patient (case report) experiencing sICH after IVT due to underlying glioblastoma multiforme. In two other studies, 1 patient suffered from gastrointestinal hemorrhage and 2 patients from cervical epidural hemorrhage $[16,17]$. In one of these patients, it is unclear whether spinal hematoma was pre-existing, hence the cause of stroke-like symptoms and not a complication of IVT [16]. In the other case, the hematoma may also have existed before IVT, as suggested by the clinical presentation with acute shoulder pain and face-sparing hemiparesis [17]. Though our data suggest that the risk of hemorrhage seems to be low, a direct comparison of the bleeding risk attributable to IVT in SM versus IS in a large data set is warranted. Furthermore, caution is needed when interpreting these findings as the low complication risk may be valid only for experienced stroke centers.

Some limitations should be considered in this study. The sample size of the SM group was small, thus the analysis is prone to type II errors. We therefore included a meta-analysis of published SM series to better ascertain the risk of hemorrhage in patients with SM after IVT with the caveat that the definition of SM and SICH was not identical in all studies. Further support for low sICH risks in patients without IS is derived from studies that assessed intravenous r-tPA for acute myocardial infarction. The sICH risk in these patients is less than $1 \%$ despite the addition of intravenous heparin to the treatment protocol [25]. Another limitation is the use of the NIHSS for assessing the neurological outcome at discharge from the hospital. While the NIHSS is applicable to IS, its value for assessing the symptoms of conditions other than stroke is limited.

In conclusion, our study suggests that the pattern of clinical deficits measured by the NIHSS differs between SM and IS, whereas common stroke predictors such as age and vascular risk factors were similarly distributed in both groups.

\section{Acknowledgements}

The authors thank Ulrike Held and Reto Kofmehl at Horten Centre for Patient Oriented Research, University of Zurich, for their help in statistical analysis.

\section{Disclosure Statement}

The authors report no disclosures.

\section{References}

1 Hacke W, Donnan G, Fieschi C, et al: Association of outcome with early stroke treatment: pooled analysis of ATLANTIS, ECASS, and NINDS rt-PA stroke trials. Lancet 2004;363:768-774.

-2 Lyden P, Brott T, Tilley B, et al: Improved reliability of the NIH Stroke Scale using video training. NINDS TPA Stroke Study Group. Stroke 1994;25:2220-2226.

- 3 Engelter ST, Reichhart M, Sekoranja L, et al: Thrombolysis in stroke patients aged 80 years and older: Swiss survey of IV thrombolysis. Neurology 2005;65:1795-1798.

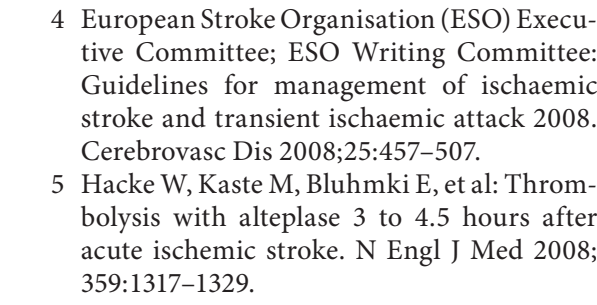
tive Committee; ESO Writing Committee: Guidelines for management of ischaemic stroke and transient ischaemic attack 2008 Cerebrovasc Dis 2008;25:457-507. bolysis with alteplase 3 to 4.5 hours afte 359:1317-1329.

Eur Neurol 2012;68:344-349

\footnotetext{
Tissue plasminogen activator for acute ischemic stroke. The National Institute of Neurological Disorders and Stroke rt-PA Stroke Study Group. N Engl J Med 1995;333:15811587.

7 van Swieten JC, Koudstaal PJ, Visser MC, Schouten HJ, van Gijn J: Interobserver agreement for the assessment of handicap in stroke patients. Stroke 1988;19:604-607.

-8 Scott PA, Silbergleit R: Misdiagnosis of stroke in tissue plasminogen activator-treated patients: characteristics and outcomes. Ann Emerg Med 2003;42:611-618.
} 
9 Mouradian MS, Rodgers J, Kashmere J, et al: Can rt-PA be administered to the wrong patient? Two patients with somatoform disorder. Can J Neurol Sci 2004;31:99-101.

10 Grimm SA, DeAngelis LM: Intratumoral hemorrhage after thrombolysis in a patient with glioblastoma multiforme. Neurology 2007;69:936.

11 Martinez-Ramirez S VA, Querol L, Marquie M, Alcolea D, Martinez-Hernandez E, Marti-Vilalta JL, Marti-Fabregas J: Frequency and outcome of patients wrongly treated with intravenous thrombolysis. Cerebrovasc Dis 2008;25:27.

12 Winkler DT, Fluri F, Fuhr P, et al: Thrombolysis in stroke mimics: frequency, clinical characteristics, and outcome. Stroke 2009; 40:1522-1525.

13 Uchino K, Massaro L, Hammer MD: Transient ischemic attack after tissue plasminogen activator: aborted stroke or unnecessary stroke therapy? Cerebrovasc Dis 2010;29:5761.
14 Chernyshev OY, Martin-Schild S, Albright KC, et al: Safety of tPA in stroke mimics and neuroimaging-negative cerebral ischemia. Neurology 2010;74:1340-1345.

15 Kern R, Förster A, Griebe M, et al: Thrombolysis has a low complication rate in patients with stroke mimics. Cerebrovasc Dis 2010;29(suppl 2):61.

16 Falcao F, Canhao P, Melo T: Stroke mimics in rtPA-treated patients. Cerebrovasc Dis 2009 27(suppl 6):48

17 Knaissi N, Derex L, Cho TH, Marnet D, Nig hoghossian N: The risk of thrombolysis in 'stroke mimics': a case report. Neurol Sci 2011;32:973-975.

18 Chen Y, Bogosavljevic V, Leys D, Jovanovic D, Beslac-Bumbasirevic L, Lucas C: Intravenous thrombolytic therapy in patients with stroke mimics: baseline characteristics and safety profile. Eur J Neurol 2011;18:12461250.

19 Giraldo EA, Khalid A, Zand R: Safety of Intravenous Thrombolysis within $4.5 \mathrm{~h}$ of symptom onset in patients with negative post-treatment stroke imaging for cerebral infarction. Neurocrit Care 2011;15:76-79.
20 Tsivgoulis G, Alexandrov AV, Chang J, et al: Safety and outcomes of intravenous thrombolysis in stroke mimics: a 6-year, singlecare center study and a pooled analysis of reported series. Stroke 2011;42:1771-1774.

21 Ferro JM, Massaro AR, Mas JL: Aetiological diagnosis of ischaemic stroke in young adults. Lancet Neurol 2010;9:1085-1096.

22 Hauf M, Slotboom J, Nirkko A, von Bredow F, Ozdoba C, Wiest R: Cortical regional hyperperfusion in nonconvulsive status epilepticus measured by dynamic brain perfusion CT. Am J Neuroradiol 2009;30:693-698.

23 Masterson K, Vargas MI, Delavelle J: Postictal deficit mimicking stroke: role of perfusion CT. J Neuroradiol 2009;36:48-51.

24 Gelfand JM, Wintermark M, Josephson SA: Cerebral perfusion-CT patterns following seizure. Eur J Neurol 2010;17:594-601.

25 An international randomized trial comparing four thrombolytic strategies for acute myocardial infarction. The GUSTO investigators. N Engl J Med 1993;329:673-682. 